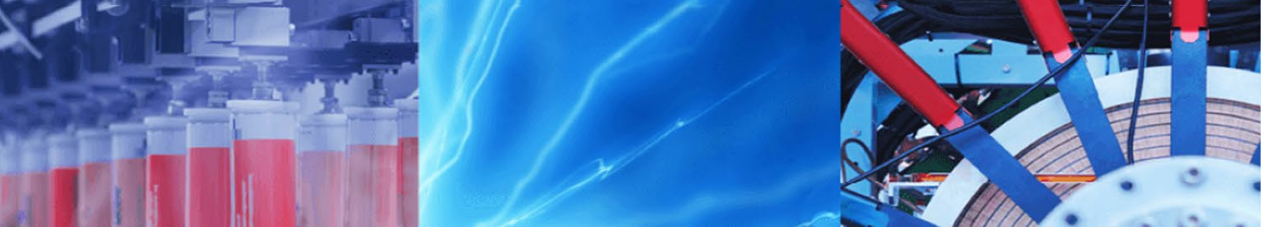

Research Article

\title{
New designed software to deconvolute the thermoluminescence glow-curves
}

\author{
Mohamed El-Kinawy ${ }^{1} \cdot$ Hassan F. El-Nashar ${ }^{1} \cdot$ Nabil El-Faramawy $^{1}$
}

C) Springer Nature Switzerland AG 2019

\begin{abstract}
Within the framework of the one trap-one recombination (OTOR) level model, a new software was designed to deconvolute the thermoluminescence glow-curves. The computer code was written considering deconvolution equations obtained, without any approximations, from the (OTOR) model with free parameters TM, IM, and $\varepsilon$. The code can also use the original equations of the OTOR model with free parameters $s, \varepsilon, n_{o^{\prime}}$ and $\beta$. The new software was applied to deconvolute glow curves measured from irradiated in-house prepared $\mathrm{Al}_{2} \mathrm{O}_{3}$ doped with $\mathrm{Cr}_{2} \mathrm{O}_{3}$ dosimeters. The consequences of the deconvolution analyses of the reference glow curves agree with those reported in the literature.
\end{abstract}

Keywords Glow curve · OTOR model Thermoluminescence $\cdot$ Deconvolution · Kinetic parameters $\cdot \mathrm{Al}_{2} \mathrm{O}_{3}$ dosimeters

\section{Introduction}

Thermoluminescence $(\mathrm{TL})$ is the thermally stimulated emission of light from insulator or semiconductor materials that absorb energy from radiation [1]. TL phenomenon requires the thermoluminescent material to possess impurities or defects. The simplest model to describe the TL phenomenon is the OTOR model. OTOR model assumes the existence of two kinds of localized states. One of the two states functions as an electron trap and the other as a hole trap (or a recombination center) [e.g. 2, 3]. In TL, thermal energy is used to release trapped electrons from their traps to the conduction band. Upon minimizing the internal energy the free electrons migrate from the conduction band to lower energy states. This transition may be radiative which causes the glow observed from the material. According to the OTOR model, this radiative transition results in the formation of a glow curve composing of a single peak. However, the glow curve measured from TL materials looks complicated due to the existence of multi-electron and hole traps. In the OTOR model, the measured glow curve is considered to be produced by summing up individual glow peaks. Each glow peak is a result of a certain electron trap. To analyze the obtained glow curve, computerized glow-curve deconvolution (CGCD) is the most used method [4].

CGCD analysis is used to find the separate glow-peaks of a composite TL glow-curve and the kinetic parameters (trap depth, frequency factor, and kinetic order) of each peak. Based on several models and approximations, many computer programs have been developed for TL glowcurve analysis [5, 6].

The aim of the current study is to consider mathematical deconvolution equations, obtained without approximations, from the OTOR model. A new software was designed to deconvolute TL glow curves using the obtained deconvolution equations. To test the new deconvolution equations and the developed code, it was, firstly, examined by analyzing the reference glow curves of GLOCANIN program $[7,8]$. Secondly, the code was used to deconvolute measured TL glow curves of proposed dosimeters, which are made of $\mathrm{Al}_{2} \mathrm{O}_{3}$ samples doped with $\mathrm{Cr}_{2} \mathrm{O}_{3}$ of different concentrations [9].

Nabil El-Faramawy, nabil_elfaramawi1@sci.asu.edu.eg| 'Department of Physics, Faculty of Science, Ain Shams University, 11566 Abbassia, Cairo, Egypt.

SN Applied Sciences (2019) 1:834 | https://doi.org/10.1007/s42452-019-0876-7

Received: 6 May 2019 / Accepted: 2 July 2019 / Published online: 5 July 2019 


\section{Method}

According to the OTOR level model, there exist two expressions describing the intensity of a TL glow peak corresponding to two kinetic orders. The intensity of a first order kinetics peak [10] is given by

$I(T)=s n_{0} \exp \left(-\frac{\varepsilon}{k T}\right) \exp \left(-\frac{s}{\beta} \int_{T_{0}}^{T} \exp \left(-\frac{\varepsilon}{k T^{\prime}}\right) d T^{\prime}\right)$

where $\mathrm{s}$ is the frequency factor $\left(\mathrm{s}^{-1}\right), \mathrm{n}_{0}$ is the initial concentration of trapped electrons $\left(\mathrm{cm}^{-3}\right), \epsilon$ is the activation energy $(\mathrm{eV}), \mathrm{k}$ is Boltzmann constant $\left(\mathrm{J} \mathrm{K}^{-1}\right), \mathrm{T}$ is the absolute temperature $(K), \beta$ is the heating rate and $T_{0}$ is the initial temperature of the heating profile. For the second and general order kinetics, the intensity of photons produced from the TL material is written as $[11,12]$

$I(T)=S n_{0}^{b} \exp \left(-\frac{\varepsilon}{k T}\right)\left[1+\frac{S n_{0}^{b-1}(b-1)}{\beta} \int_{T_{0}}^{T} \exp \left(-\frac{\varepsilon}{k T^{\prime}}\right) d T^{\prime}\right]^{-\frac{b}{b-1}}$

where $S=s^{\prime}$ for the second order kinetics model once $b=2$. While for the general order model $S=s^{\prime \prime}$ and $1<b<2$. It can be noticed, from Eqs. (1) and (2), that the intensity of the glow peak depends upon the integral

$$
\begin{aligned}
F_{o}\left(T, \varepsilon, T_{0}\right)= & \int_{T_{0}}^{T} \exp \left(-\frac{\varepsilon}{k T^{\prime}}\right) d T^{\prime}=T \exp \left(-\frac{\varepsilon}{k T}\right) \\
& -T_{0} \exp \left(-\frac{\varepsilon}{k T_{0}}\right)+\frac{\varepsilon}{k}\left(\mathrm{Ei}\left(-\frac{\varepsilon}{k T}\right)-\operatorname{Ei}\left(-\frac{\varepsilon}{k T_{0}}\right)\right)
\end{aligned}
$$

which is solved analytically in references [13-15], where $\mathrm{Ei}(-\mathrm{x})$, is the well-known exponential integral function [14] with $x>0$. Thus, utilizing the exact solution of the integral, the deconvolution equations obtained from the OTOR level model in terms of the maximum intensity and maximum temperature [16] can be reformed. A computer code, using the MATLAB package, is developed to study the deconvolution of TL glow curves. Moreover, the code is devoted to employing the current deconvolution equations because the commercial software uses approximations in regard to the solution of the integral (Eq. 3). Another point to consider is that current computers have limited precision and available accuracies that do not exceed 32 bits. Consequently, including many terms in the exact solution of the integral (Eq. 3) ensures a good level of accuracy in calculations and, in general, permits overcoming the limitations of computer resources.

\subsection{First order kinetics model}

The intensity, according to Eq. (1), for the first order kinetics model can be written, using the solution of the integral (3), by inserting Eq. (3) into (1), as:

$I(T)=s n_{0} \exp \left(-\frac{\varepsilon}{k T}\right) \exp \left[-\frac{s}{\beta}\left(T \exp \left(-\frac{\varepsilon}{k T}\right)+\frac{\varepsilon}{k}\left(\operatorname{Ei}\left(-\frac{\varepsilon}{k T}\right)\right)\right)\right]$

Equation (4) gives the intensity, for $T>T_{0}$, of a first order TL peak and it is a function of $s, \epsilon, T, \beta$, and $T_{0}$. In CGCD analysis it is desired to obtain a form of the intensity $I$ as a function of maximum TL intensity and its corresponding temperature (i.e. $I_{M}$ and $T_{M}$ respectively) [16] as well as the energy $\epsilon$. To achieve this goal in accordance with the last sentence, the conditions at maximum intensity are used $[16,17]$ and they lead to:

$$
\begin{aligned}
I= & I_{M} \exp \left(\frac{\varepsilon}{k T}\left(\frac{T-T_{M}}{T_{M}}\right)\right) \exp \left(\frac{\varepsilon}{k T_{M}^{2}}\right. \\
& \left.\exp \left(\frac{\varepsilon}{k T_{M}}\right)\left(F\left(T_{M}, \varepsilon\right)-F(T, \varepsilon)\right)\right)
\end{aligned}
$$

where $F(T, \varepsilon)$ and $F\left(T_{M}, \varepsilon\right)$ are defined, respectively, as

$F(T, \varepsilon)=T \exp \left(-\frac{\varepsilon}{k T}\right)+\frac{\varepsilon}{k}\left(\mathrm{Ei}\left(-\frac{\varepsilon}{k T}\right)\right)$

and

$F\left(T_{M}, \varepsilon\right)=T_{M} \exp \left(-\frac{\varepsilon}{k T_{M}}\right)+\frac{\varepsilon}{k}\left(\mathrm{Ei}\left(-\frac{\varepsilon}{k T_{M}}\right)\right)$

Equation (5) is now ready to be used for a glow curve analysis. For the first order kinetics

$s=\frac{\beta \varepsilon}{k T_{M}^{2}} \exp \left(\frac{\varepsilon}{k T_{M}}\right)$

and

$I_{M}=\frac{n_{0} \beta \varepsilon}{k T_{M}^{2}} \exp \left(-\frac{\varepsilon}{k T_{M}^{2}} \exp \left(\frac{\varepsilon}{k T_{M}}\right) F_{o}\left(T_{M}, \varepsilon, T_{0}\right)\right)$

Equations 8 and 9 are used to calculate the frequency factor and the initial concentration of trapped electrons $n_{0}$ of a first order glow peak.

\subsection{Second- and general-order kinetics model}

The second order kinetics model results as soon as $b=2$ in Eq. (2) while the general order kinetics is obtained when $1<b<2$. Therefore, the intensity of photons 
produced from the TL material obeying a second order process is given by (11)

$I(T)=s^{\prime} n_{0}^{2} \exp \left(-\frac{\varepsilon}{k T}\right)\left[1+\frac{s^{\prime} n_{o}}{\beta}\left(T \exp \left(-\frac{\varepsilon}{k T}\right)+\frac{\varepsilon}{k}\left(\mathrm{Ei}\left(-\frac{\varepsilon}{k T}\right)\right)\right)\right]^{-2}$

with $\mathrm{n}_{0}, \epsilon, T, \beta$, and $\mathrm{T}_{0}$ being the same parameters defined earlier. The quantity s' is the pre-exponential factor $\left(\mathrm{cm}^{3}\right.$ $\left.\mathrm{s}^{-1}\right)$. The conditions at maximum intensity, when they are used, lead to

$I(T)=I_{M} \exp \left(\frac{\varepsilon}{k T}\left(\frac{T-T_{M}}{T_{M}}\right)\right)\left[1+\frac{\varepsilon\left(F(T, \varepsilon)-F\left(T_{M}, \varepsilon\right)\right)}{2 k T_{M}^{2} \exp \left(-\frac{\varepsilon}{k T_{M}}\right)}\right]^{-2}$

Equations (10) and (11) are employed in the deconvolution analysis according to the second order kinetics for $T>T_{0}$. The two functions $F(T, \epsilon)$ and $F\left(T_{M}, \epsilon\right)$ are given by the two Eqs. (6) and (7), respectively. For the second order kinetics,

$s^{\prime}=\frac{1}{n}\left(\frac{\beta \varepsilon}{2 k T_{M}^{2} \exp \left(-\frac{\varepsilon}{k T_{M}}\right)-\varepsilon F_{o}\left(T_{M}, \varepsilon, T_{0}\right)}\right)$

and

$$
\begin{aligned}
I_{M}= & n_{0} \frac{\beta \varepsilon}{2 k T_{M}^{2} \exp \left(-\frac{\varepsilon}{k T_{M}}\right)-\varepsilon F_{o}\left(T_{M}, \varepsilon, T_{o}\right)} \exp \left(-\frac{\varepsilon}{k T_{M}}\right) \\
& \times\left[1+\frac{\varepsilon F_{o}\left(T_{M}, \varepsilon, T_{o}\right)}{2 k T_{M}^{2} \exp \left(-\frac{\varepsilon}{k T_{M}}\right)-\varepsilon F_{o}\left(T_{M}, \varepsilon, T_{o}\right)}\right]^{-2}
\end{aligned}
$$

where $s=s^{\prime} n_{o}$.

In a similar way to first order kinetics, Eqs. (12) and (13) are used to calculate the pre-exponential factor $s^{\prime}$ and $n_{0}$ of a second order glow peak.

In a similar manner, once the values of $b$ are $1<b<2$, a general order kinetics formula for the intensity of light emitted from the TL material, as suggested by May and Partridge (12), is obtained. In this case, this intensity is written as

$$
\begin{aligned}
I(T)= & s^{\prime \prime} n_{0}^{b} \exp \left(-\frac{\varepsilon}{k T}\right)\left[1+\frac{s^{\prime \prime} n_{0}^{b-1}(b-1)}{\beta}\left(T \exp \left(-\frac{\varepsilon}{k T}\right)\right.\right. \\
& \left.\left.+\frac{\varepsilon}{k}\left(\operatorname{Ei}\left(-\frac{\varepsilon}{k T}\right)\right)\right)\right]^{-\frac{b}{b-1}}
\end{aligned}
$$

where $s^{\prime \prime}$ is a pre-exponential factor and it has the dimensions $\mathrm{cm}^{3(\mathrm{~b}-1)} \mathrm{s}^{-1}$. Upon using the conditions at maximum intensity, it can be obtained

$$
\begin{aligned}
I(T)= & \mathrm{I}_{M} \exp \left(\frac{\varepsilon}{k T}\left(\frac{T-T_{M}}{T_{M}}\right)\right) \\
& \times\left[1+\frac{\varepsilon(b-1)\left(F(T, \varepsilon)-F\left(T_{M}, \varepsilon\right)\right)}{k T_{M}^{2} b \exp \left(-\frac{\varepsilon}{k T_{M}}\right)}\right]^{\frac{-b}{b-1}}
\end{aligned}
$$

For the general order

$s^{\prime \prime}=\frac{1}{n_{o}^{b-1}}\left(\frac{\beta \varepsilon}{k b_{M}^{2} \exp \left(-\frac{\varepsilon}{k T_{M}}\right)-\varepsilon(b-1) F_{o}\left(T_{M}, \varepsilon, T_{o}\right)}\right)$

and

$$
\begin{aligned}
I_{M}= & \frac{n_{0} \beta \varepsilon}{k b T_{M}^{2} \exp \left(-\frac{\varepsilon}{k T_{M}}\right)-\varepsilon(b-1) F_{o}\left(T_{M}, \varepsilon, T_{0}\right)} \exp \left(-\frac{\varepsilon}{k T_{M}}\right) \\
& \times\left(1+\frac{\varepsilon(b-1) F_{o}\left(T_{M}, \varepsilon, T_{0}\right)}{k b T_{M}^{2} \exp \left(-\frac{\varepsilon}{k T_{M}}\right)-\varepsilon(b-1) F_{o}\left(T_{M}, \varepsilon, T_{0}\right)}\right)^{\frac{-b}{b-1}}
\end{aligned}
$$

where $s=s^{\prime \prime} n_{o}^{b-1}$.

For general order kinetics, Eqs. (16) and (17) can be used to calculate the pre-exponential factor $s^{\prime \prime}$ and $n_{0}$ of a general order glow peak.

Equations (15) and (17) are used to achieve the TL glow curve analysis in view of the general order kinetics, for T>T0. Equations (6) and (7), respectively, represent the two terms $F(T, \epsilon)$ and $F\left(T_{M}, \epsilon\right)$.

\subsection{Other forms}

Based on the OTOR model, an expression for the intensity of a single peak is obtained in terms of the Lambert function $[18,19]$, given by

$I(T)=I_{M} \frac{W\left[\exp \left(Z_{M}\right)\right]+W\left[\exp \left(Z_{M}\right)\right]^{2}}{W[\exp (Z)]+W[\exp (Z)]^{2}} \exp \left(-\frac{E}{k T} \frac{T_{M}-T}{T_{M}}\right)$

where

$$
Z_{M}=\frac{R}{1-R}-\ln \left(\frac{1-R}{R}\right)+E \frac{\exp \left(\frac{E}{k T_{M}}\right)}{k T_{M}^{2}\left(1-1.05 R^{1.26}\right)} F\left(T_{M}, E\right)
$$


$Z=\frac{R}{1-R}-\ln \left(\frac{1-R}{R}\right)+E \frac{\exp \left(\frac{E}{k T_{M}}\right)}{k T_{M}^{2}\left(1-1.05 R^{1.26}\right)} F(T, E)$

where $F(T, E)$ and $F\left(T_{M}, E\right)$ given by Eqs. (6) and (7), respectively. In Eqs. (19) and (20) $R=A_{n} / A_{m}$ where $A_{n}$ and $A_{m}$ are the trapping and recombination probability coefficients. In case of $A_{n}<A_{m}, R$ in the OTOR model resembles the kinetic order $\mathrm{b}$ in the GOK model. And $\mathrm{W}(\mathrm{x})$ is the Lambert function. The value of $s$ (OTOR model) is calculated by using the peak maximum condition and it is given by the equation:

$s=\frac{k T_{M}^{2}}{\beta \varepsilon} \exp \left(\frac{\varepsilon}{k T}\right)\left[\frac{1}{1-R} \frac{1+2 W\left[\exp \left(Z_{M}\right)\right]}{1+W\left[\exp \left(Z_{M}\right)\right]^{2}}\right]^{-1}$

\subsection{MATLAB code}

The developed MATLAB code employs Eqs. (4), (5), (10), (11), (14), (15) and (18) to be used in the process of TL glow curve deconvolution analysis. Therefore, the user chooses the desired equation for TL deconvolution in the first step. The MATLAB code utilizes Nelder-Mead, Levenberg-Marquardt, and Trust-Region algorithms. Any of these nonlinear optimization algorithms are used to perform TL glow curve deconvolution with considerable efficiency, and they are accurate in determining the peak parameters for experimental overlapping TL glow curves. As a second step, the user chooses the fitting algorithm. It is well known that the non-linear fitting is hugely dependent on the choice of the initial guess of the fitting parameters. Thus, in the third step, the user determines the number of peaks. Then he/she ignites the algorithm with an initial guess of each peak parameters. As declared in almost all optimization algorithms, they stop when they reach specific accuracy by changing the optimization parameters regardless of the physical meaning of the resultant final parameters. Therefore, the user is given the privilege of fixing some parameters if the solver fails to get acceptable physical parameters.

\section{Material}

GLOCANIN represents standard reference glow curves that belong to the first order kinetics. It is a good candidate for testing the MATLAB code for TL glow curve deconvolution. GLOCANIN project $[7,8]$ includes ten reference glow curves (RGCs) ensuing first order kinetics model only. RGCs 1 and 2 are obtained from computer simulation [7]. Those RGCs, 1 and 2, were simulated to be composed of 1 single peak for RefGlow.001 and 4 summed peaks for
RefGlow.002 so that RefGlow.002 imitates the glow curve structure of TLD-100 [7]. The left eight RGCs are measured from samples irradiated at low and high doses [8]. Most of those eight reference glow curves show, relatively, simple glow curves composing of three overlapping peaks for RGCs $7 \& 8,4$ overlapping peaks for RGCs $3,4, \& 10$ and 5 overlapping peaks for RGCs $5 \& 6$. The most complicated RGC is number 9 which is obtained from a highly irradiated TLD-700 sample. The deconvolution of this RGC differs, in literature, not only in the number of deconvoluted peaks but also in their position and shape [8].

Second, the current deconvolution expressions are employed to analyze the experimental glow curves of samples of $\mathrm{Cr}_{2} \mathrm{O}_{3}-\mathrm{Al}_{2} \mathrm{O}_{3}$ nanoparticles doped with $\mathrm{Cr}_{2} \mathrm{O}_{3}$. A different series of $\mathrm{Cr}_{2} \mathrm{O}_{3}-\mathrm{Al}_{2} \mathrm{O}_{3}$ nanoparticles containing several molar compositions of $\mathrm{Cr}_{2} \mathrm{O}_{3}(0-5 \mathrm{wt} \%)$ are prepared by the sol-gel method. The samples are irradiated using beta rays with a dose of $3 \mathrm{~Gy}$. Then the irradiated samples are read out at a heating rate of $4 \mathrm{~K} / \mathrm{s}$. The TL measurements were achieved by RISO-TL-DA-12 reader with a built-in $90 \mathrm{Sr} / 90 \mathrm{Y} \beta$-source with a rate of $3.33 \mathrm{mGy} / \mathrm{s}$. The samples were read out at the Institute of Radiation Protection, Helmholtz research centre, Munich, Germany [9].

\subsection{Test of the computer code}

The CGCD analysis of the GLOCANIN RGCs of Bos et al. [7, 8] is performed by first order kinetics. The performance of

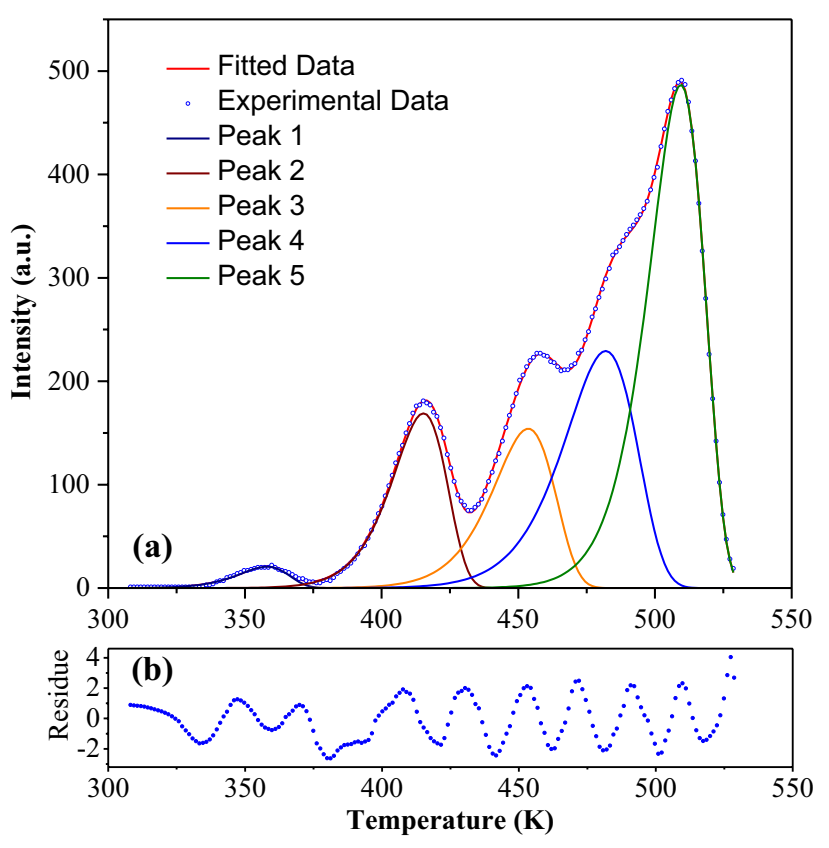

Fig. 1 The deconvolution of the RefGlow.005 glow-curve of the GLOCANIN project $[7,8]$. The FOM value is $0.6793 \%$ 


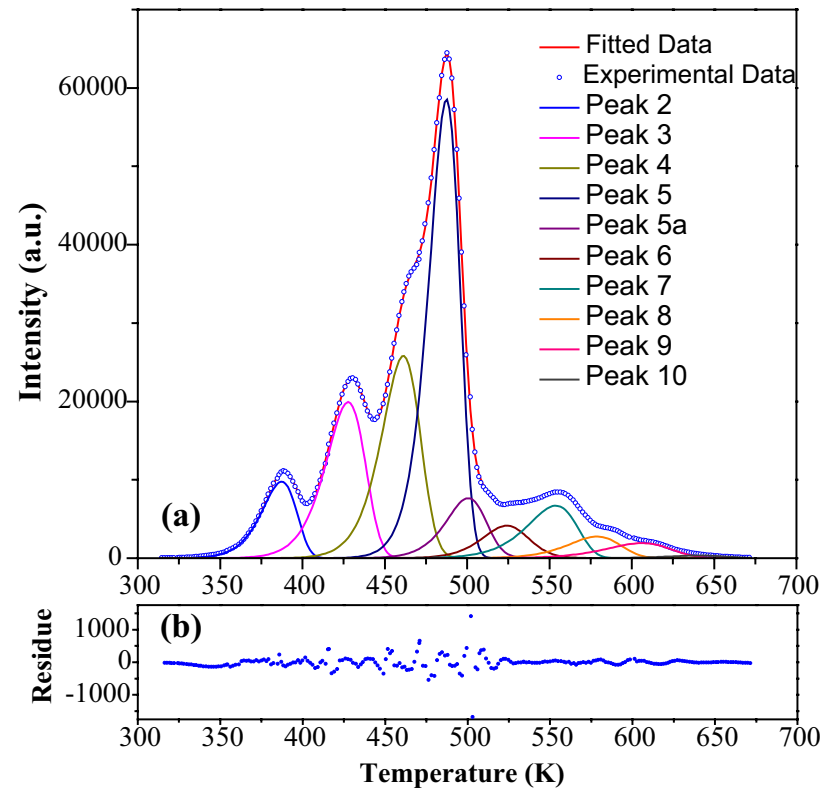

Fig. 2 The deconvolution of the RefGlow.009 glow-curve of the GLOCANIN project $[7,8]$. The FOM value is $0.8453 \%$

fitting is tested with the figure of merit (FOM) as defined in Ref. [20]. Figures 1 and 2 show the CGCD analysis of the measured reference glow curves 5 and 9 , respectively. The top graphs of Figs. 1 and 2 indicate the matching between the measured signal and the fitted curves. The lower patterns in both Figs. 1 and 2 demonstrate the residue (the difference between the data of the GLOCANIN curves and the fitted).

The values of the activation energy obtained using the developed code, employing the current expressions, for each glow-peak, are compared with the real values (for RefGlow.001 and 002) or the mean values (for RefGlow.003-010) obtained in references [7, 8]. The deviation of the obtained activation energies of the 10 reference glow curves in $[7,8]$ from the real (or mean) values and the obtained FOM values are shown in Table 1.

There exist no standard results or projects to test the second and general order equations. Therefore, synthetic second and general order glow curves were generated by convoluting peaks using the proper equations (Eqs. 10 and 2) of the second and general order kinetic equations. Then, we used the MATLAB code to deconvolute the synthetic glow curves. The FOM values obtained in the case of the currently proposed functions were of the order of $10^{-5} \%$.

\subsection{Deconvolution of $\mathrm{TL}$ glow curves of $\mathrm{Al}_{2} \mathrm{O}_{3}$ doped with $\mathrm{Cr}_{2} \mathrm{O}_{3}$}

The experimental glow curves of $\mathrm{Cr}_{2} \mathrm{O}_{3}-\mathrm{Al}_{2} \mathrm{O}_{3}$ nanoparticles were deconvoluted using the designed software. The glow-curve analysis of $\mathrm{Al}_{2} \mathrm{O}_{3}$ nanoparticles containing a molar composition of $\mathrm{Cr}_{2} \mathrm{O}_{3}$ of $1.5 \mathrm{wt} \%$, between 297 and $685 \mathrm{~K}$, is shown in Fig. 3, which reveals a deconvolution of 6 peaks. The obtained trap depths of the 6 peaks were found to be $0.625,0.691,0.801,0.949,1.006$, and $1.071 \mathrm{eV}$, respectively. The corresponding orders of kinetic parameters of those peaks were 1.57, 1.39, 1.69, $1.53,1.52$, and 1.28. Figure 4 shows the deconvolution of $\mathrm{Al}_{2} \mathrm{O}_{3}$ nanoparticles containing a molar composition of $\mathrm{Cr}_{2} \mathrm{O}_{3}$ of $5.0 \mathrm{wt} \%$, between 236 and $685 \mathrm{~K}$. The obtained trap depths of these 5 peaks were found to be 0.735 , $0.773,0.822,0.937$, and $0.942 \mathrm{eV}$, respectively. The orders of kinetic parameters of those peaks were 1.41, 1.29, 1.54, 1.55, and 1.45. A comparison between Figs. 3 and 4 indicates that $\mathrm{Al}_{2} \mathrm{O}_{3}$ nanoparticles with compositions of $1.5 \% \mathrm{Cr}_{2} \mathrm{O}_{3}$ show a higher response for beta radiation than the other compositions.
Table 1 The deviation of the activation energies values, which are obtained by the CGCD using the exact TL expressions, from those values obtained in references ( 15 , 16). The table also shows the values of FOM (\%) for each glow curve

\begin{tabular}{lllllll}
\hline File name & \multicolumn{2}{l}{ Deviation in E (eV) } & FOM (\%) \\
\cline { 2 - 6 } & Peak 1 & Peak 2 & Peak 3 & Peak 4 & Peak 5 & \\
\hline RefGlow.001 & 0 & - & - & - & - & 0.0099 \\
RefGlow.002 & - & 0.001 & 0.001 & 0.001 & 0 & 0.0099 \\
RefGlow.003 & - & -0.036 & -0.019 & -0.016 & 0.0154 & 0.9541 \\
RefGlow.004 & - & -0.034 & -0.011 & -0.037 & -0.05 & 0.9815 \\
RefGlow.005 & - & -0.012 & 0.004 & -0.057 & 0.023 & 0.6793 \\
RefGlow.006 & - & -0.034 & -0.018 & -0.033 & -0.028 & 0.8723 \\
RefGlow.007 & - & - & -0.066 & 0.003 & -0.046 & 1.0348 \\
RefGlow.008 & - & - & -0.042 & -0.014 & -0.015 & 0.9048 \\
RefGlow.009 & - & -0.029 & 0.005 & -0.045 & 0.042 & 0.8453 \\
RefGlow.010 & - & -0.065 & 0.057 & -0.062 & -0.014 & 1.2874 \\
\hline
\end{tabular}




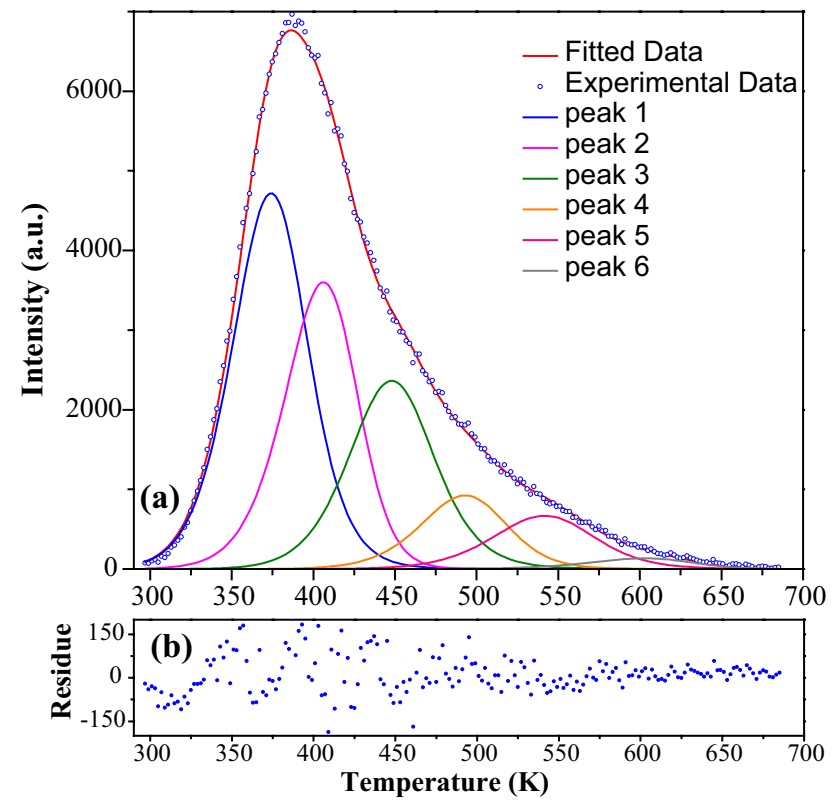

Fig. 3 Fitting $\mathrm{TL}$ glow curve of $\mathrm{Cr}_{2} \mathrm{O}_{3}-\mathrm{Al}_{2} \mathrm{O}_{3}$ nanoparticles containing molar compositions of $\mathrm{Cr}_{2} \mathrm{O}_{3}$ of $1.5 \mathrm{wt} \%$. The FOM value is $1.1372 \%$
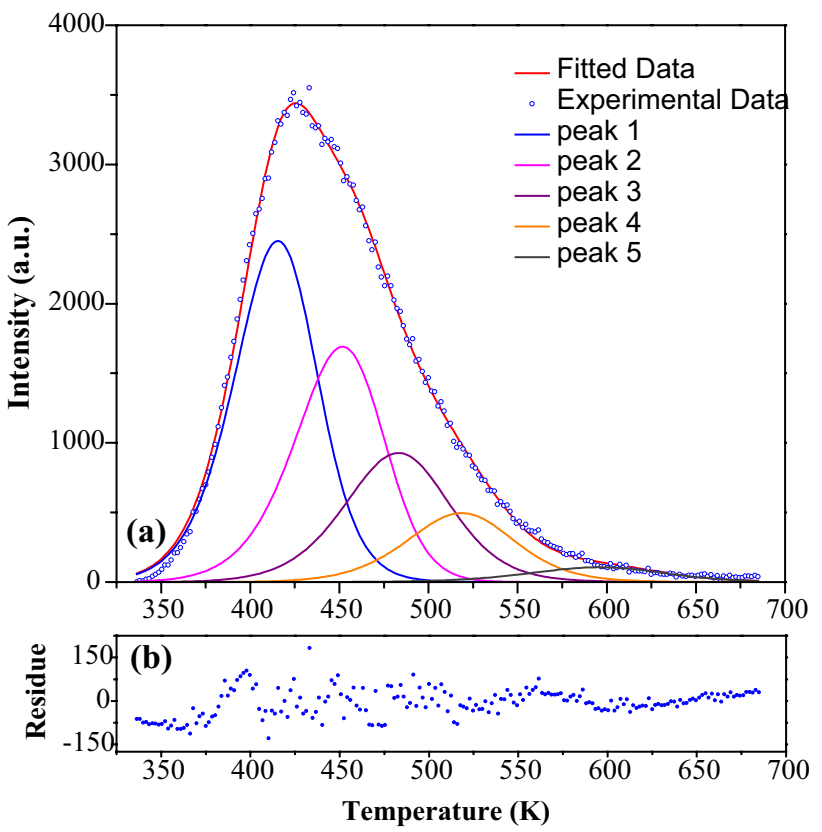

Fig. 4 Fitting $\mathrm{TL}$ glow curve of $\mathrm{Cr}_{2} \mathrm{O}_{3}-\mathrm{Al}_{2} \mathrm{O}_{3}$ nanoparticles containing molar compositions of $\mathrm{Cr}_{2} \mathrm{O}_{3}$ of 5.0 wt\%. The FOM value is $1.9521 \%$

\section{Conclusions}

Within the frame of the OTOR level model, the exact equations of the intensities of a TL glow peaks as functions of temperature for different kinetics orders were considered. A new software was developed to, computationally, deconvolute TL glow curves. The CGCD analysis, using the equations of the first order kinetics, is achieved for the GLOCANIN reference glow-curves of references $[7,8]$. The capability of the current TL deconvolution expressions to get accurate analyses of the TL glow-curves with low values of FOM is shown. The deconvolution equations of the second and general order kinetics were tested using preconstructed glow curves (synthetics), and the obtained results reveal excellent agreements with low FOM values.

The current deconvolution equation of the general kinetic order was employed to deconvolute the TL glow curves of $\mathrm{Al}_{2} \mathrm{O}_{3}$ doped with different concentrations of $\mathrm{Cr}_{2} \mathrm{O}_{3}$. It was found that $\mathrm{Al}_{2} \mathrm{O}_{3}-\mathrm{Cr}_{2} \mathrm{O}_{3}$ nanoparticles with compositions of $1.5 \% \mathrm{Cr}_{2} \mathrm{O}_{3}$ exhibit the highest response to beta radiation. $\mathrm{Al}_{2} \mathrm{O}_{3}-\mathrm{Cr}_{2} \mathrm{O}_{3}$ nanoparticles material with a concentration of $1.5 \%$ of $\mathrm{Cr}_{2} \mathrm{O}_{3}$ represented a promising dosimeter for beta radiation. It also exemplifies a promising dosimeter for gamma radiation at low doses. More details about $\mathrm{Al}_{2} \mathrm{O}_{3}-\mathrm{Cr}_{2} \mathrm{O}_{3}$ nanoparticles material with a concentration of $1.5 \%$ of $\mathrm{Cr}_{2} \mathrm{O}_{3}$ need more studies when it is irradiated with gamma radiation.

Acknowledgement The authors are thankful to professor G. Kitis for providing the reference glow curves of GLOCANIN project.

\section{Compliance with ethical standards}

Conflict of interest The authors declare that they have no conflict of interest.

\section{References}

1. McKeever SWS (1985) Thermoluminescence of solids. Cambridge University Press, London

2. Adirovitch El (1956) La formule de Becquerel et la loi élémentaire du déclin de la luminescence des phosphores cristallins. J Phys Rad 17:705-707

3. Halperin A, Braner AA (1960) Evaluation of thermal activation energies from glow curves. Phys Rev 117:408-415

4. Horowitz YS, Yossian D (1995) Computerised glow curve deconvolution: application to thermoluminescence dosimetry. Radiat Prot Dosim 60:293-295

5. Horowitz YS, Mosowitch M (1986) LiF-TLD in the microgray dose range via computerised glow curve deconvolution and background smoothing. Radiat Prot Dosim 17:337-342

6. Delgado A, Gomez-Ros JM (2001) Computerised glow curve analysis: a tool for routine thermoluminescence dosimetry. Radiat Prot Dosim 96:127-132 
7. Bos AJJ, Piters TM, Gomez-Ros JM, Delgado A (1993) An intercomparison of glow curve analysis computer programs I. Synthetic glow curves. Radiat Prot Dosim 47:473-477

8. Bos AJJ, Piters TM, Gomez-Ros JM, Delgado A (1994) An intercomparison of glow curve analysis computer programs II. Measured glow curves. Radiat Prot Dosim 51:257-264

9. Ahmed M, Salah A, Ashour A, Hafez H, El-Faramawy N (2018) Dosimetric properties of $\mathrm{Cr}$ doped $\mathrm{Al}_{2} \mathrm{O}_{3}$ nanophosphors. J Lumin 196:449-454

10. Randall JT, Wilkins MHT (1945) Phosphorence and electron traps I the study of trap distributions. Proc R Soc A 184:365-389

11. Garlick GFJ, Gibson AF (1948) The electron trap mechanism of luminescence in sulphide and silicate phosphors. Proc Phys Soc 60:574-590

12. May CE, Partridge JA (1964) Thermoluminescent kinetics of alpha-irradiated alkali halides. J Chem Soc 40:1401-1409

13. Leonard I, Grossweiner A (1953) Note on the analysis of first order glow curves. J Appl Phys 24:1306-1307

14. Erdelyi E (1954) Tables of integral transforms, vol II. McGraw Hill, New York

15. Gradshteyn IS (2007) Ryzhik IM "table of integrals, series, and products", 7th edn. Academic Press, Amsterdam
16. Kitis G, Gomez-Ros JM, Tuyn JWN (1998) Thermoluminescence glow-curve deconvolution functions for first, second and general order of kinetics. J Phys D Appl Phys 31:2636-2641

17. Furetta $C$ (2010) Handbook of thermoluminescence, 2nd edn. World Scientific Publishing Co. Pte. Ltd., Singapore

18. Kitis G, Vlachos ND (2013) General semi-analytical expressions for $T L, O S L$ and other luminescence stimulation modes derived from the OTOR model using the Lambert W-function. Radiat Meas 48:47-54

19. Sadek AM, Eissa HM, Basha AM, Carinou E, Askounis P, Kitis G (2015) The deconvolution of thermoluminescence glow-curves using general expressions derived from the one trap-one recombination (OTOR) level model. Appl Radiat Isot 95:214-221

20. Balian HG, Eddy NW (1977) Figure-of-merit (FOM), an improved criterion over the normalized Chi squared test for assessing goodness-of-fit of gamma-ray spectral peaks. Nucl Instrum Methods 145:389-395

Publisher's Note Springer Nature remains neutral with regard to jurisdictional claims in published maps and institutional affiliations. 